\title{
Structure and Dynamics of a Temperature-Sensitive Hydrogel
}

Published as part of The Journal of Physical Chemistry virtual special issue "Dor Ben-Amotz Festschrift". Eliane P. van Dam, Hongbo Yuan, Paul H. J. Kouwer, and Huib J. Bakker*

Cite This: J. Phys. Chem. B 2021, 125, 8219-8224

Read Online

ACCESS 1

Џlll Metrics \& More

Article Recommendations

Supporting Information

ABSTRACT: Polyisocyanotripeptides (TriPIC) are biomimetic polymers which consist of a $\beta$-helical backbone stabilized by hydrogen bonds between amide groups. Their oligoethylene glycol side chains give aqueous TriPIC solutions a thermoresponsive behavior: at $50{ }^{\circ} \mathrm{C}$ the solution becomes a hydrogel. In this paper we study the molecular structure and water dynamics of TriPIC aqueous solutions while undergoing gelation using FT-IR spectroscopy and polarization-resolved femtosecond infrared spectroscopy (fs-IR). We find evidence that the oligoethylene glycol side chains trap part of the water molecules upon gel formation, and we propose that the interaction between the oligoethylene glycol side chains and water plays an essential role in the bundling of the polymers and thus in the formation of a hydrogel.

\section{INTRODUCTION}

Many biological gels consist of polymers that assemble in helical structures, which give them stiffness and stability. ${ }^{1,2}$ These biological hydrogels often show a special mechanical responsiveness that plays an essential role in cellular processes. $^{3-5}$ It is interesting to mimic biological hydrogels with gels based on synthetic polymers, as these synthetic gels will find many applications. ${ }^{6,7}$ Rowan and co-workers developed a biomimetic hydrogel, based on polyisocyanopeptides (PICs), that not only possesses a similar mechanical responsiveness as many biological gels but also shows thermoresponsive behavior. ${ }^{8}$

PICs consist of a helical backbone that is responsible for the gel stiffness and that can be chemically modified to add a targeted functionality to the polymer. PICs adopt a $4_{1} \beta$-helical conformation (four repeating units per turn). This conformation is stabilized by a $\beta$-sheet-like hydrogen-bond network between the amide groups of monomers $n$ and $n+$ 4. ${ }^{9,10}$ The stiff, helical backbone of the TriPIC polymer chain contains three adjacent alanine groups (see Figure 1) and is stabilized by two parallel hydrogen-bonding arrays between the amide groups, which provide the polymer with thermal stability. In a previous study using FT-IR, we showed that the amide group closest to the helical backbone is inaccessible to the water solvent. ${ }^{11}$

When the polyisocyanopeptides are grafted with ethylene glycol side chains, they exhibit a thermoresponsive behavior that causes the polymer to form a gel at elevated temperatures. ${ }^{8,12}$ These synthetic gels have been shown to form a network of semiflexible bundles and to mimic biological gels.

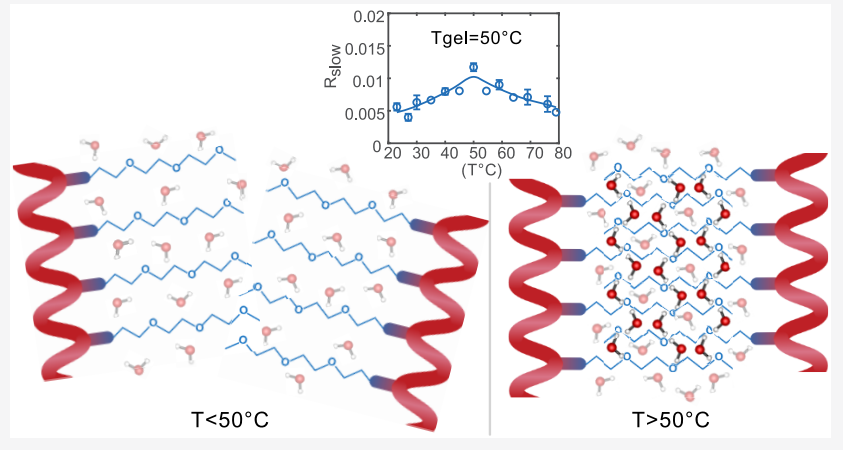

These gels have been studied with several microscopy techniques, small-angle X-ray scattering, and rheology measurements. Nevertheless, the molecular mechanism of the gelation process of PICs remains largely unknown. ${ }^{12,13}$

To fully understand the gelation process of PICs and to be able to design other biomimetic hydrogels, it is essential to know their spatial conformation and intermolecular interactions. Here we use polarization-resolved femtosecond infrared spectroscopy to study the dynamics of the water molecules in TriPIC polymer solutions and how these dynamics change when the temperature is increased above the gelation temperature.

\section{EXPERIMENTAL SECTION}

Sample Preparation. TriPIC monomer and polymer molecules were synthesized according to the protocol given in ref 11 . The monomer and TriPIC polymer solutions for polarization-resolved femtosecond infrared spectroscopic studies were prepared by dissolving the monomer or polymer in $4 \%$ $\mathrm{D}_{2} \mathrm{O}$ (99.9\% D, Cambridge Isotope Laboratories) in $\mathrm{H}_{2} \mathrm{O}$ (ultrapure Milli-Q grade) and by stirring the solution for $24 \mathrm{~h}$ at $4{ }^{\circ} \mathrm{C}$. Temperature-dependent pump-probe measurements were performed by placing the sample inside a sample cell that

Received: April 7, 2021

Revised: June 25, 2021

Published: July 19, 2021 
A<smiles>C=[N+]([O-])C(C)C(=O)NC(C)C(=O)NC(C)C(=O)OCCOCCOCCOC</smiles>

B<smiles>COCCOCCOCCOC(=O)C(C)NC(=O)C(C)NC(=O)C(C)N=C(C)C(C)(C)C</smiles>

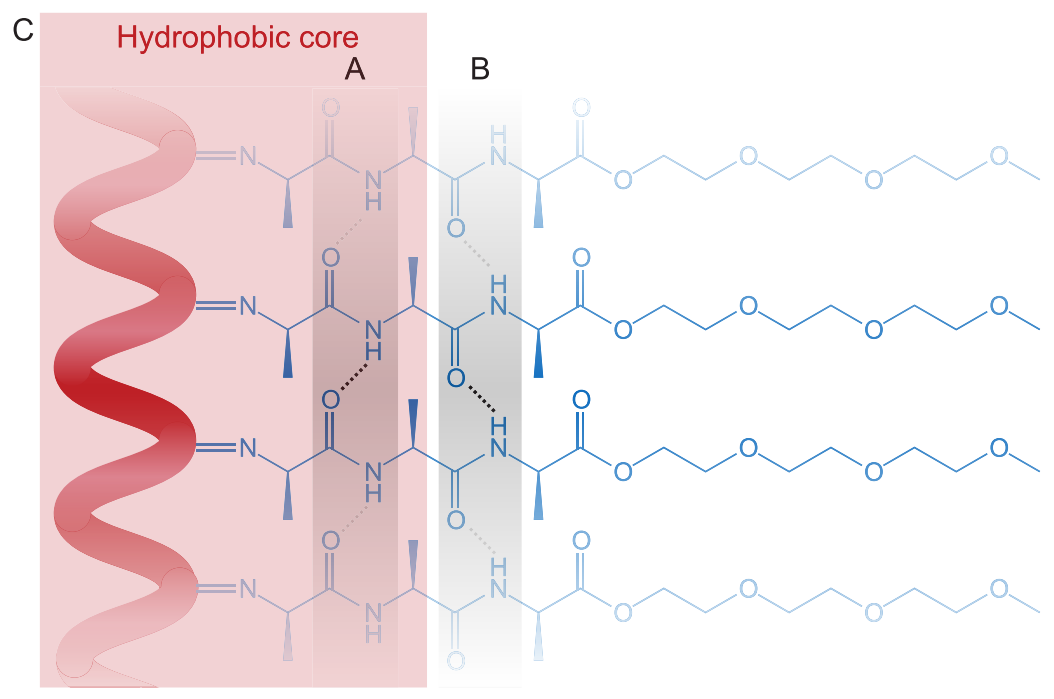

Figure 1. (A) Molecular structure of the TriPIC monomer. (B) Molecular structure of the TriPIC polymer. (C) Cartoon of the $\beta$-helical structure of TriPIC and the hydrogen-bonded network between the amide groups, with the water inaccessible hydrophobic core (in red) and the stabilizing hydrogen bonds (in gray).

was closed by calcium fluoride windows and by heating this cell with a Peltier element. After the temperature was set to a new value, the measurement was started after a stabilization time of $5 \mathrm{~min}$ to allow the sample to adapt to the new temperature and to form a gel for temperatures above the gelation temperature (gelation occurs within a few minutes).

Pump-Probe Spectroscopy. We study the reorientation dynamics of water molecules by pumping and probing the OD stretch vibration of $\mathrm{HDO}$ molecules in monomer and polymer samples containing isotopically diluted water (obtained by adding $2 \% \mathrm{D}_{2} \mathrm{O}$ to $\mathrm{H}_{2} \mathrm{O}$ ). We excite the OD stretch vibration with an intense laser pulse with a frequency centered around $2500 \mathrm{~cm}^{-1}$. We probe the induced absorption changes using a second, weaker laser pulse, also centered around $2500 \mathrm{~cm}^{-1}$. The polarization of the probe pulse is either parallel or perpendicular to that of the pump pulse, and we thus measure the pump-induced transient absorption changes in both parallel $\left(\Delta \alpha_{\|}(\omega, t)\right)$ and perpendicular $\left(\Delta \alpha_{\perp}(\omega, t)\right)$ pumpprobe polarization configurations. After excitation with the pump pulse the absorption change will be largest when measured with a probing pulse with a polarization direction that is parallel to that of the pump because the pump pulse excites vibrations that have their transition dipole moment oriented along the polarization direction most efficiently. At longer delay times between pump and probe pulses, the difference between the parallel and perpendicular absorption changes decreases because of the reorientation of the HDO molecules. From the parallel and perpendicular signals, we construct the isotropic signal $\Delta \alpha_{\text {iso }}$ :

$$
\Delta \alpha_{\text {iso }}=\frac{1}{3}\left(\Delta \alpha_{\|}(\omega, t)+2 \Delta \alpha_{\perp}(\omega, t)\right)
$$

This isotropic signal decays with the vibrational relaxation time and is not affected by reorientational relaxation. After correcting the measured transient absorption changes for the contribution of the ingrowing heating signal, ${ }^{14}$ we can construct the anisotropy $R$ of the transient absorption signal associated with the excitation of the OD vibrations:

$$
R=\frac{\Delta \alpha_{\|}(\omega, t)-\Delta \alpha_{\perp}(\omega, t)}{\Delta \alpha_{\|}(\omega, t)+2 \Delta \alpha_{\perp}(\omega, t)}
$$

This signal decays because of the reorientation of the HDO molecules and is independent of the vibrational relaxation. A detailed description of the experimental setup used for the experiment is given elsewhere. ${ }^{15}$

\section{RESULTS AND DISCUSSION}

The transient absorption spectra of $40 \mathrm{mg} / \mathrm{mL}$ TriPIC monomer and polymer solutions in isotopically diluted water are shown in Figure 2. These spectra show the transient absorption changes of the OD stretch vibration of the HDO molecules at different time delays. For both solutions, we observe a bleaching signal near $2500 \mathrm{~cm}^{-1}$ at early time delays, which we assign to bleaching of the ground state and stimulated emission out of the first excited vibrational state back to the ground state. With increasing delay time this signal decays due to vibrational relaxation. The vibrational relaxation leads to local heating of the sample so that at later delay times 

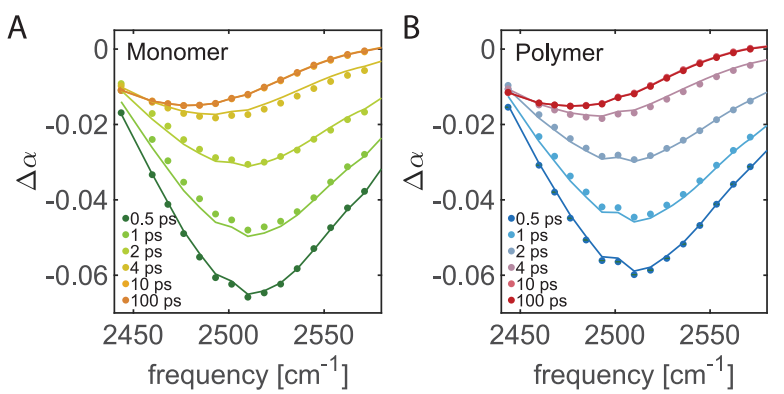

Figure 2. Isotropic transient absorption changes of the OD stretch vibration of HDO molecules for solutions of (A) $40 \mathrm{mg} / \mathrm{mL}$ monomer in isotopically diluted water and (B) $40 \mathrm{mg} / \mathrm{mL}$ TriPIC polymer in isotopically diluted water for different picosecond delay times. The solid lines result from a fit to the model described in the text.

the signal is formed by the difference between the linear absorption at an elevated temperature and linear absorption spectrum at room temperature. To extract the anisotropy of the signal component corresponding to the excitation of the OD stretch vibration, we need to correct the transient absorption signals for the latter thermal effect. To this purpose, we fit the transient absorption spectra with a kinetic model describing the vibrational relaxation of the OD stretch vibration and the ingrowing heating contribution. The results of this fit are shown as the solid lines in Figure 2.

Concentration Dependence. In Figure 3 we show the anisotropy decay of the OD stretch vibration of $\mathrm{HDO}$
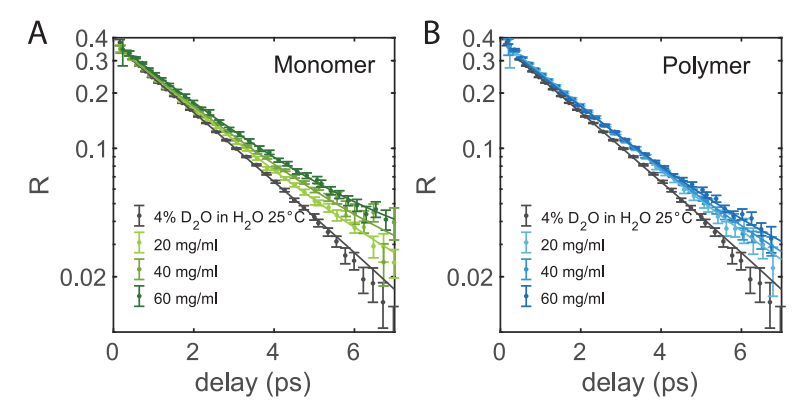

Figure 3. (A) Anisotropy decay as a function of delay time of monomer solutions in isotopically diluted water for different concentrations at room temperature. (B) Anisotropy decay as a function of delay time of TriPIC polymer solutions in isotopically diluted water for different concentrations at room temperature. The solid lines are fits to an exponential function with an offset.

measured for pure isotopically diluted water and for TriPIC monomer and polymer solutions at different concentrations at room temperature. For pure isotopically diluted water, we find that the anisotropy decay can be fitted well with an exponential function with a (reorientation) time constant of 2.3 ps. For solutions of TriPIC monomer or polymer, we observe a similar exponential decay, but also an additional much slower component. We thus fit the anisotropy decay of the TriPIC monomer and polymer solutions with an exponential function and an offset, $R=R_{0} \mathrm{e}^{-t / \tau_{\mathrm{r}}}+R_{\text {slow }}$. We find for all TriPIC monomer and polymer solutions a reorientation time $\tau_{\mathrm{r}}$ of 2.4 ps, similar to the reorientation time of neat isotopically diluted water. This finding indicates that most of the water molecules in TriPIC monomer and polymer solutions reorient in a similar manner as in bulk water and that a small fraction of the water molecules reorient on a much longer time scale, probably as a result of their interaction with the TriPIC monomers and polymers.

In Figure 4 we present the offset $R_{\text {slow }}$ as a function of solute concentration $c_{\text {solute }}$ for both the TriPIC monomer and the

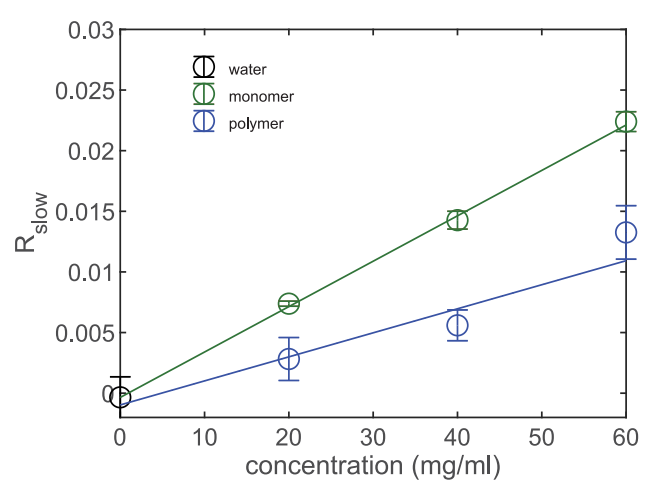

Figure 4. Offset $R_{\text {slow }}$ of the anisotropy decay as a function of concentration for the monomer (green) and TriPIC polymer (blue) and isotopically diluted water (gray) at room temperature. The solid lines are fits to a linear function.

polymer. It is clearly seen that $R_{\text {slow }}$ is larger for the monomer than for the polymer below the gelation temperature. The linear dependence of $R_{\text {slow }}$ on the solute concentration indicates that the slowly reorienting water hydroxyl groups are associated with water molecules that are in the direct vicinity of the solute. Based on earlier studies of the effects of solutes on the reorientation dynamics of water, ${ }^{16,17}$ these slow water molecules likely interact with the hydrophobic groups or the polyethylene glycol tails of the monomer and TriPIC polymer.

From the slope $a$ of the linear fit $\left(R_{\text {slow }}=a c_{\text {solute }}+b\right)$, we calculate the number of slowly reorienting water molecules $N_{\text {slow }}$ per monomer molecule (for the monomer solutions) or repeating unit (for the polymer solutions) by using the following expression:

$$
N_{\text {slow }}=\frac{a M_{\mathrm{w}}}{0.4} \times 110.514
$$

where $M_{\mathrm{w}}$ is the molecular weight of the TriPIC repeating unit (to change the units from $\mathrm{mg} / \mathrm{mL}$ to molal), and the factor 110.514 represents the number of moles of hydroxyl groups in a kilogram of water (for $4 \% \mathrm{D}_{2} \mathrm{O}$ in $\mathrm{H}_{2} \mathrm{O}$ ). The factor of 0.4 in the denominator is added because this is the maximum value of the anisotropy parameter if all water hydroxyl groups would be slow.

We find that at room temperature the monomer slows down $40 \pm 2$ water hydroxyl groups per molecule while the polymer slows down $21 \pm 3$ water hydroxyl groups per repeating unit. The difference between the amount of slowly reorienting water hydroxyl groups between the monomer and polymer can be explained from the conformation of the polymer. As we showed in our previous study, for the polymer the inner amide, amide $\mathrm{A}$, is not accessible for the solvent, meaning that the water molecules are not interacting with the inner core of the polymer. ${ }^{11}$ In contrast, the monomer is completely water accessible. Interestingly, this difference in solvent accessibility cannot fully explain the difference of $\sim 20$ slowly rotating water $\mathrm{OH}$ groups between the monomer and the polymer. In previous work it was found that one $\mathrm{CH}$ bond slows down the 
rotation of on average 1.25 water $\mathrm{OH}$ groups. ${ }^{16}$ Upon polymer formation, eight $\mathrm{CH}$ groups become solvent inaccessible, which is thus expected to lead to a decrease of the number of slowly reorienting water $\mathrm{OH}$ groups by $\sim 10$. Recently, Ensing et al. showed that polyethylene glycol molecules slow down the reorientation of water molecules not only because of the interaction of water molecules with the hydrophobic groups, but also because of the strong interactions of water with the partially charged polyethylene glycol oxygen atoms. ${ }^{17}$ In the polymer the glycol chains of different monomer units are closely spaced, with the result that the oxygen atoms of and the hydrophobic parts of the glycol chains are less exposed to water than in the case of the unbound monomers. As a result, fewer water molecules are slowed in their reorientation for the polymer than for the monomer, thus explaining why the difference in number of slow water hydroxyl groups between monomer and polymer is larger than expected from only the exclusion of water from the core of the polymer.

Temperature Dependence. To investigate the role of water in the gelation process of the TriPIC polymer, we measure the water reorientation dynamics as a function of temperature for both the TriPIC monomer and the polymer solutions. The anisotropic decays are displayed in Figure 5. In
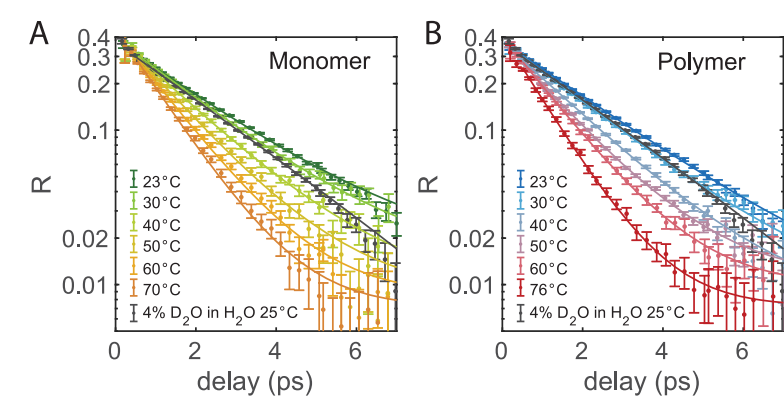

Figure 5. (A) Anisotropy decay as a function of delay time of $40 \mathrm{mg} /$ $\mathrm{mL}$ monomer solutions in isotopically diluted water for different temperatures. (B) Anisotropy decay as a function of delay time of 40 $\mathrm{mg} / \mathrm{mL}$ TriPIC polymer solutions in isotopically diluted water for different temperatures. The solid lines are fits to an exponential function with an offset.

both cases, the reorientation time becomes faster with increasing temperature. This decrease in reorientation time is also observed for pure isotopically diluted water. ${ }^{18}$ To analyze the data, we fitted the reorientation time constants measured for pure isotopically diluted water at different temperatures with an Arrhenius expression (see the Supporting Information). The temperature-dependent reorientation times resulting from this fitted Arrhenius expression were then used as the exponential time constants in fits of the measured anisotropy decays of the TriPIC monomer and polymer solutions. We thus fit these anisotropy decays with an exponential function plus an offset, like we did for the concentration-dependent measurements at room temperature. The results of these fits are presented as the solid lines in Figure 5.

In Figure 6, we show the offset $R_{\text {slow }}$ as a function of temperature for both the TriPIC monomer and polymer. For the monomer, we observe a monotone decrease of $R_{\text {slow }}$ with increasing temperature. This finding agrees with the dynamics of water measured for proteins at different temperatures. ${ }^{19}$ Previous dielectric relaxation measurements and NMR experiments on small amphiphilic molecules also showed a similar decrease in slowly reorienting water molecules with increasing

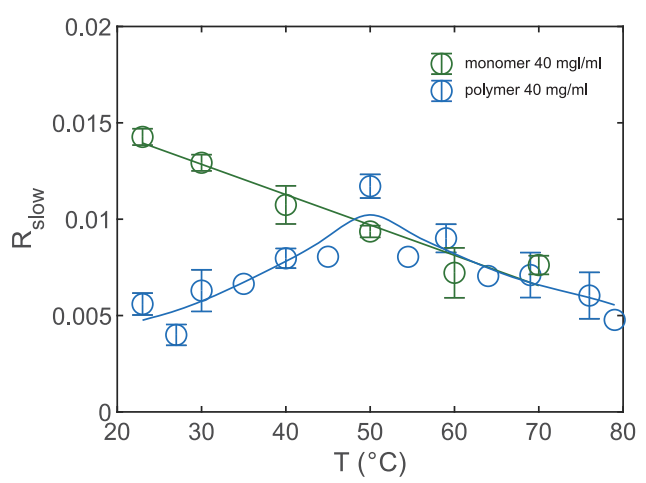

Figure 6. Offset $R_{\text {slow }}$ of the anisotropy decay as a function of temperature for the monomer (green) and TriPIC polymer (blue) at $40 \mathrm{mg} / \mathrm{mL}$. The solid lines are guides to the eye.

temperature. $^{20,21}$ This decrease of the number of slowly reorienting hydroxyl groups can be explained from the fact that the hydrogen-bond network becomes more dynamic and less structured at higher temperatures. ${ }^{20}$ For the polymer, we observe a quite different temperature dependence. At temperatures below the gelation temperature of $50{ }^{\circ} \mathrm{C}, R_{\text {slow }}$ increases with increasing temperature, until a maximum $R_{\text {slow }}$ at $50{ }^{\circ} \mathrm{C}$ is reached after which the offset decreases. The difference in temperature dependence between the monomer and the polymer is striking, and the fact that the turning point for the polymer occurs exactly at the gelation temperature $T_{\text {gel }}=$ $50{ }^{\circ} \mathrm{C}$ suggests that for the polymer the change in water dynamics with temperature is directly related to the gelation process of the TriPIC polymer solution.

It should be noted that the increase in slowly reorienting water molecules below $50{ }^{\circ} \mathrm{C}$ cannot be attributed to an increase of the solvent accessibility of the core of the polymer with increasing temperature, as we found that the solvent accessibility of this core does not change significantly upon heating of the solution. ${ }^{11}$ We show FT-IR spectra of the solid polymer below and above gelation temperature in Figure SI2 to illustrate this point. We see that the amide II band, dominated by the $\mathrm{NH}$ bending vibration, stays present in $\mathrm{D}_{2} \mathrm{O}$ solvent, at temperatures of $65{ }^{\circ} \mathrm{C}$, well above the gelation temperature. We thus attribute the increase of the fraction of slowly reorienting water hydroxyl groups to the binding of an increasing number of water molecules to the polyethylene glycol side chains of different polymers. We propose that the trapping of water molecules in between the negatively charged oxygen atoms of polyethylene glycol side chains of different polymers plays a crucial role in the bundling of the polymers. In this picture the water molecules act as a glue that binds polymers together to form bundles and a polymer network, which ultimately at $50{ }^{\circ} \mathrm{C}$ leads to hydrogel formation. With increasing temperature, the amount of water molecules that act as a glue builds up, reaching a maximum at $T_{\text {gel }}$. For TriPIC monomers, the polyethylene glycol chains are already quite strongly exposed to water at room temperature, and changing the temperature does not lead to a significant change of this interaction.

At temperatures $>50{ }^{\circ} \mathrm{C}$ the fraction of slow water molecules is similar for the TriPIC polymer and the monomer. At these higher temperatures, the amount of slowly reorienting water molecules decreases with increasing temperature. This behavior is similar to what we observe for the monomer over the whole temperature range and has been observed 
before for proteins and amphiphilic molecules. ${ }^{19-21}$ Although the amounts of slowly reorienting water molecules for the monomer and the polymer are similar at these temperatures, this does not mean that their hydration would be the same. For TriPIC polymers, water is excluded from the core, leading to a smaller number of water molecules that is slowed by the central part of the molecule, but apparently at temperatures $>50{ }^{\circ} \mathrm{C}$, this lower number is compensated by the water molecules that are slowed as a result of their trapping between the polyethylene glycol chains in the polymer bundles, probably because within these bundles water molecules can easily bind to two ether oxygen atoms simultaneously, which leads to a stronger slowing down of their reorientation. ${ }^{17}$ Above the gel formation temperature, the amount of water molecules being tightly bound between the polyethylene glycol tails does not change further with temperature, and the reorientation behavior of the water molecules in between and outside the polymer chains is the same as is observed for other amphiphilic molecules and polymer systems. ${ }^{19-21}$

The increase of the slow water fraction upon gelation of TriPIC polymers forms an interesting contrast with the effect of gelation on the slow water fraction in hyaluronic acid hydrogels. ${ }^{15}$ For aqueous solutions of hyaluronic acid (which forms a hydrogel at $\mathrm{pH} 2.5$ ), we showed that the slow fraction is in fact decreasing upon gelation. The disparity between the observations for these two hydrogels can be explained from the different nature of the interactions that govern the hydrogel formation. In the case of hyaluronic acid, the network formation underlying the formation of the hydrogel is the result of the formation of direct hydrogen bonds between the carboxylate groups and the amide groups of the hyaluronic acid molecules, thus not involving water molecules. ${ }^{22}$ Water molecules are in fact expelled from the region between the polymer chains when forming these intermolecular hydrogen bonds. For TriPIC polymer solutions, the network formation underlying the hydrogel formation likely relies on the binding of bridging water molecules in between the polyethylene glycol chains, thus leading to cross-linking of these chains. As these water molecules will show a very slow reorientation, TriPIC gel formation is accompanied by an increase of the slow water fraction.

The above mechanism of gel formation of the TriPIC polymers implies that this formation involves counteracting contributions of the entropy of the water molecules. The bundling of the polyethylene glycol chains will lead to a reduction of the configurational space of the water molecules that remain hydrogen bonded to the glycol chains, which represents a negative contribution to the water entropy. In addition, the bundling involves a reduction of the configurational space of the polymer chains themselves and probably also an increase in enthalpy, as part of the water molecules that were strongly hydrogen-bonded to the glycol chains in the solution phase are expelled from the polymer chains upon gel formation. Apparently, above the gel formation temperature, these losses in entropy and increase in enthalpy are sufficiently compensated by the large gain in entropy of the vast majority of water molecules outside the polymer chains.

\section{CONCLUSIONS}

In summary, we studied the molecular structure and water reorientation dynamics of TriPIC monomer and polymer solutions with temperature-dependent polarization-resolved femtosecond infrared spectroscopy. We find that below the gelation temperature TriPIC polymers slow down fewer water molecules per unit than TriPIC monomers.

With increasing temperature, the fraction of water molecules that are slowed by the TriPIC polymers increases, opposite to what is observed for the monomer and for other solute molecules in water. We explain this increase from the enhanced binding of water molecules to the polyethylene glycol side chains. We propose that this enhanced binding may play a role in the bundling of the polymers, in particular when the water molecule donates two strong hydrogen bonds to two negatively charged oxygen atoms of polyethylene glycol side chains that belong to different polymers. The water molecules thus likely act as a glue that binds the polymers together, leading to an extended polymer network and thereby to the formation of a hydrogel.

At the gelation temperature of $50{ }^{\circ} \mathrm{C}$, the number of trapped water molecules saturates. When the temperature is increased above the gelation temperature, the temperature dependence of the number of slowed-down water molecules becomes normal; that is, the number of slow water molecules decreases with increasing temperature, similar as is observed for solutions of TriPIC monomers.

\section{ASSOCIATED CONTENT}

\section{Supporting Information}

The Supporting Information is available free of charge at https://pubs.acs.org/doi/10.1021/acs.jpcb.1c03121.

Additional ATR spectra, temperature-dependent anisotropy of isotopically diluted water (PDF)

\section{AUTHOR INFORMATION}

\section{Corresponding Author}

Huib J. Bakker - AMOLF, 1098 XG Amsterdam, The Netherlands; 이이.org/0000-0003-1564-5314; Email: h.bakker@amolf.nl

\section{Authors}

Eliane P. van Dam - AMOLF, 1098 XG Amsterdam, The Netherlands; 이이이.org/0000-0002-3051-8922

Hongbo Yuan - Institute of Biophysics, Hebei University of Technology, Tianjin 300401, P. R. China; Institute for Molecules and Materials, Radboud University, $6525 \mathrm{AJ}$ Nijmegen, The Netherlands

Paul H. J. Kouwer - Institute for Molecules and Materials, Radboud University, 6525 AJ Nijmegen, The Netherlands;

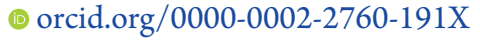

Complete contact information is available at:

https://pubs.acs.org/10.1021/acs.jpcb.1c03121

\section{Notes}

The authors declare no competing financial interest.

\section{ACKNOWLEDGMENTS}

This work is part of the research program of The Netherlands Organization for Scientific Research (NWO) and of the Industrial Partnership Programme Hybrid Soft Materials that is carried out under an agreement between Unilever Research and Development B.V. and The Netherlands Organization for Scientific Research (NWO). The work was performed at the research institute AMOLF. 


\section{REFERENCES}

(1) Giannotti, M. I.; Vancso, G. J. Interrogation of Single Synthetic Polymer Chains and Polysaccharides by AFM-Based Force Spectroscopy. ChemPhysChem 2007, 8 (16), 2290-2307.

(2) Van Buul, A. M.; Schwartz, E.; Brocorens, P.; Koepf, M.; Beljonne, D.; Maan, J. C.; Christianen, P. C. M.; Kouwer, P. H. J.; Nolte, R. J. M.; Engelkamp, H.; Blank, K.; Rowan, A. E. Stiffness versus Architecture of Single Helical Polyisocyanopeptides. Chem. Sci. 2013, 4 (6), 2357-2363.

(3) Discher, D. E.; Janmey, P.; Wang, Y. L. Tissue Cells Feel and Respond to the Stiffness of Their Substrate. Science (Washington, DC, U. S.) 2005, 310 (5751), 1139-1143.

(4) Engler, A. J.; Sen, S.; Sweeney, H. L.; Discher, D. E. Matrix Elasticity Directs Stem Cell Lineage Specification. Cell 2006, 126 (4), 677-689.

(5) Das, R. K.; Gocheva, V.; Hammink, R.; Zouani, O. F.; Rowan, A. E. Stress-Stiffening-Mediated Stem-Cell Commitment Switch in Soft Responsive Hydrogels. Nat. Mater. 2016, 15 (3), 318-325.

(6) Zhang, C.; Mcadams, D. A.; Grunlan, J. C. Nano/MicroManufacturing of Bioinspired Materials: A Review of Methods to Mimic Natural Structures. Adv. Mater. 2016, 28 (30), 6292-6321.

(7) Li, Q.; Barrett, D. G.; Messersmith, P. B.; Holten-Andersen, N. Controlling Hydrogel Mechanics via Bio-Inspired Polymer-Nanoparticle Bond Dynamics. ACS Nano 2016, 10 (1), 1317-1324.

(8) Kouwer, P. H. J.; Koepf, M.; Le Sage, V. a A.; Jaspers, M.; van Buul, A. M.; Eksteen-Akeroyd, Z. H.; Woltinge, T.; Schwartz, E.; Kitto, H. J.; Hoogenboom, R.; Picken, S. J.; Nolte, R. J. M.; Mendes, E.; Rowan, A. E. Responsive Biomimetic Networks from Polyisocyanopeptide Hydrogels. Nature 2013, 493 (7434), 651-655.

(9) Millich, F.; Baker, G. K. Polyisonitriles. III. Synthesis and Racemization of Optically Active Poly ( $\alpha$-Phenylethylisonitrile). Macromolecules 1969, 2 (2), 122-128.

(10) Cornelissen, J. J. L. M.; Donners, J. J. J. M.; De Gelder, R.; Graswinckel, W. S.; Metselaar, G. A.; Rowan, A. E.; Sommerdijk, N. A. J. M.; Nolte, R. J. M. $\beta$-Helical Polymers from Isocyanopeptides. Science (Washington, DC, U. S.) 2001, 293 (5530), 676-680.

(11) Yuan, H.; Xu, J.; Van Dam, E. P.; Giubertoni, G.; Rezus, Y. L. A.; Hammink, R.; Bakker, H. J.; Zhan, Y.; Rowan, A. E.; Xing, C.; Kouwer, P. H. J. Strategies to Increase the Thermal Stability of Truly Biomimetic Hydrogels: Combining Hydrophobicity and Directed Hydrogen Bonding. Macromolecules 2017, 50 (22), 9058.

(12) Jaspers, M.; Dennison, M.; Mabesoone, M. F. J.; MacKintosh, F. C.; Rowan, A. E.; Kouwer, P. H. J. Ultra-Responsive Soft Matter from Strain-Stiffening Hydrogels. Nat. Commun. 2014, DOI: $10.1038 /$ ncomms6808.

(13) Jaspers, M.; Pape, A. C. H.; Voets, I. K.; Rowan, A. E.; Portale, G.; Kouwer, P. H. J. Bundle Formation in Biomimetic Hydrogels. Biomacromolecules 2016, 17 (8), 2642-2649.

(14) Rezus, Y. L. A.; Bakker, H. J. On the Orientational Relaxation of HDO in Liquid Water. J. Chem. Phys. 2005, 123 (11), 114502.

(15) van Dam, E. P.; Giubertoni, G.; Burla, F.; Koenderink, G. H.; Bakker, H. J. Hyaluronan Biopolymers Release Water upon PHInduced Gelation. Phys. Chem. Chem. Phys. 2020, 22, 8667.

(16) Rezus, Y. L. A.; Bakker, H. J. Observation of Immobilized Water Molecules around Hydrophobic Groups. Phys. Rev. Lett. 2007, 99 (14), 1-4.

(17) Ensing, B.; Tiwari, A.; Tros, M.; Hunger, J.; Domingos, S. R.; Pérez, C.; Smits, G.; Bonn, M.; Bonn, D.; Woutersen, S. On the Origin of the Extremely Different Solubilities of Polyethers in Water. Nat. Commun. 2019, 10 (1), 1-8.

(18) Tielrooij, K. J.; Petersen, C.; Rezus, Y. L. A.; Bakker, H. J. Reorientation of $\mathrm{HDO}$ in Liquid $\mathrm{H}_{2} \mathrm{O}$ at Different Temperatures: Comparison of First and Second Order Correlation Functions. Chem. Phys. Lett. 2009, 471 (1-3), 71-74.

(19) Groot, C. C. M. Dynamics of Water Interacting with Biomolecules, 2018.

(20) Tielrooij, K. J.; Hunger, J.; Buchner, R.; Bonn, M.; Bakker, H. J. Influence of Concentration and Temperature on the Dynamics of
Water in the Hydrophobic Hydration Shell of Tetramethylurea. J. Am. Chem. Soc. 2010, 132 (44), 15671-15678.

(21) Qvist, J.; Halle, B. Thermal Signature of Hydrophobic Hydration Dynamics. J. Am. Chem. Soc. 2008, 130 (31), 1034510353.

(22) Giubertoni, G.; Burla, F.; Martinez-Torres, C.; Dutta, B.; Pletikapic, G.; Pelan, E.; Rezus, Y. L. A.; Koenderink, G. H.; Bakker, H. J. Molecular Origin of the Elastic State of Aqueous Hyaluronic Acid. J. Phys. Chem. B 2019, 123 (14), 3043-3049. 